\title{
Intervenção nutricional sobre medidas antropométricas e glicemia de jejum de pacientes diabéticos
}

\section{$\left(\int\right.$}

Nutritional intervention on anthropometric

measurements and fasting glycemia

of diabetic patients

Júnia Maria GERALDO²

Rita de Cássia Gonçalves ALFENAS ${ }^{1}$

Raquel Duarte Moreira ALVES²

Vannina de Freitas SALLES²

Valéria Maria Vitarelli QUEIROZ ${ }^{3}$

Maria Christina Barros BITENCOURT ${ }^{3}$

\section{RE S U M O}

\section{Objetivo}

Avaliar o alcance das atividades desenvolvidas em um projeto de atendimento nutricional, promovido pela Universidade Federal de Viçosa (MG), sobre a glicemia de jejum, medidas antropométricas e de composição corporal de indivíduos diabéticos.

\section{Métodos}

Trata-se de um estudo de coorte, no qual pacientes diabéticos, recrutados do Projeto de Atendimento Nutricional a Diabéticos da Terceira Idade, da Universidade Federal de Viçosa, participaram de reuniões quinzenais, nas quais foram abordados temas relativos à ingestão de dietas saudáveis, e receberam atendimento nutricional individualizado. Os dados foram coletados a partir da aplicação de um roteiro clínico-nutricional, a 42 pacientes, contendo informações pessoais e itens referentes a: história clínica, inquérito dietético, dados antropométricos, de composição corporal e bioquímicos. Analisaram-se o peso, o índice de massa corporal, a circunferência da cintura, o percentual de gordura corporal e a glicemia de jejum antes de qualquer intervenção e após seis meses de acompanhamento. As análises estatísticas foram conduzidas utilizando-se o software Statistical Package for the Social Sciences, versão 12.5. O critério de significância estatística adotado foi $p<0,05$. As análises, antes e depois do período de intervenção, foram realizadas pelo teste de Wilcoxon.

\footnotetext{
${ }^{1}$ Universidade Federal de Viçosa,Curso de Nutrição, Departamento de Nutrição e Saúde. Av. PH Rolfs, s/n., Campus UFV, 36570-000, Viçosa, MG, Brasil. Correspondência para/Correspondence to: R.C.G. ALFENAS.

2 Acadêmicas, Universidade Federal de Viçosa, Curso de Nutrição. Viçosa, MG, Brasil.

3 Técnicas, Universidade Federal de Viçosa, Departamento de Nutrição e Saúde. Viçosa, MG. Brasil.
} 
330 | J.M. GERALDO et al.

\section{Resultados}

Observou-se redução estatisticamente significante dos valores medianos do índice de massa corporal $(p=0,032$ e 0,022), da circunferência da cintura $(p=0,036$ e 0,03) e do percentual de gordura corporal $(p=0,045$ e 0,039$)$ dos voluntários dos sexos masculino e feminino, respectivamente. Também foi observada redução estatisticamente significante na glicemia de jejum $(p=0,035)$, apesar de o valor mediano deste parâmetro ainda ter permanecido acima das recomendações.

\section{Conclusão}

Os resultados indicam que a terapia nutricional parece ter contribuído para a melhoria na adequação das medidas antropométricas e bioquímicas dos participantes, embora seja necessária a adesão ao tratamento proposto por um maior período de tempo, para que tais alterações se tornem ainda mais evidentes.

Termos de indexação: Antropometria. Avaliação nutricional. Diabetes mellitus. Dieta. Glicemia.

\section{A B S T R A C T}

\section{Objective}

The purpose of the present study was to evaluate the scope of a project of nutritional intervention, conducted by the Federal University of Viçosa (MG), on fasting glucose, anthropometric measurements and body composition of diabetic patients.

\section{Methods}

In this cohort study, diabetic patients recruited from the "Elderly Diabetics Nutritional Care" project, conducted at the Federal University of Viçosa, participated in meetings twice a month, where healthy diets were discussed and individual nutritional intervention was given. The data were collected by the application of a clinical-nutritional questionnaire to 42 patients containing personal information and information regarding clinical history, dietary intake, anthropometric measurements, body composition and biochemical data. Body weight, body mass index, waist circumference, percentage of body fat and fasting glucose of the participants were analyzed at baseline and after six months of nutritional intervention. The statistical analyses were conducted using the Statistical Package for the Social Sciences software, version 12.5. Statistical significance was set at $p<0.05$. The analyses before and after the intervention were done using the Wilcoxon test.

\section{Results}

At the end of the study, there was a significant reduction in the median body mass index ( $p=0.032$ and 0.022$)$, waist circumference $(p=0.036$ and 0.03$)$, and percentage of body fat ( $p=0.045$ and 0.039$)$ of males and females, respectively. Furthermore, there was also a significant reduction in fasting glucose $(p=0.035)$. However, the median value of this last parameter was still above the recommended value.

\section{Conclusion}

The results indicate that the nutritional therapy helped improve the anthropometric and biochemical data of the participants, although they must continue with this treatment for more time for these changes to become more evident.

Indexing terms: Athropometry. Nutritional assessment. Diabetes mellitus. Diet. Glycemia.

\section{N T R O D U Ç Ã O}

O diabetes mellitus (DM) é considerado uma doença metabólica caracterizada pela hiperglicemia, resultante do defeito da secreção e/ou ação insulínica. Sua prevalência e incidência têm aumentado consideravelmente no mundo, principalmente entre os idosos' ${ }^{1}$. Segundo a World Health Organization, em 2003 havia, aproximadamente, 150 milhões de indivíduos diabéticos no mundo, sendo previsto o dobro desse número para o ano de $2025^{2}$. 
O controle ineficiente do DM propicia uma desordem metabólica, causando alterações macrovasculares, que se iniciam prematuramente, e microvasculares, que ocorrem quando a hiperglicemia é mais freqüente ${ }^{3,4}$. Dentre as complicações, destacam-se retinopatia, nefropatia, doenças coronarianas, acidente vascular cerebral, ulcerações no pé (pé diabético) e amputação5 .

Atualmente, há grande preocupação com o elevado custo econômico e social associado ao diabetes mellitus tipo 2, fazendo com que seja necessária a implementação de ações que visem ao controle glicêmico e evitem a progressão desta enfermidade. A elaboração de políticas públicas e o desenvolvimento de atividades educativas em saúde, como as nutricionais, são estratégias eficazes na redução dos gastos relacionados à doença ${ }^{5-7}$. Além disso, a história natural do DM mostra seu caráter crônico e oligossintomático, podendo permanecer imperceptível por muitos anos. Isto comprova que a atenção primária e a prevenção devem ter prioridade, embora ações de reabilitação não devam ser negligenciadas ${ }^{4,7}$.

O tratamento da doença envolve alterações no estilo de vida, principalmente em relação aos hábitos alimentares, realização de atividade física e uso de medicamentos (hipoglicemiantes orais ou insulina) $)^{1,3,4}$.

Segundo a American Diabetes Association, a melhor estratégia nutricional para a promoção da saúde e redução do risco de doença crônica é a obtenção de nutrientes adequados a partir de uma alimentação variada, moderada e equilibrada, baseada nos pilares da Pirâmide de Alimentos ${ }^{1}$. A dieta indicada para pacientes diabéticos deve ser rica em fibras, com baixos teores de gordura saturada, sal e açúcares simples. A fibra solúvel favorece o controle de dislipidemias e da glicemia, por reduzir a absorção de colesterol e carboidratos no âmbito intestinal. Dietas moderadas em carboidratos e proteínas e de baixo teor lipídico são especialmente indicadas para portadores de diabetes ${ }^{8}$.

As medidas antropométricas e bioquímicas são de suma importância para avaliação do estado nutricional e evolução dos indivíduos diabéticos, já que auxiliam a monitorar as possíveis mudanças apresentadas e a traçar o tipo de tratamento dietético mais adequado. Dessa forma, ambas as medidas refletem a eficácia do tratamento e a adesão do paciente ao mesmo?.

Nesse contexto, o acompanhamento nutricional aos pacientes diabéticos, público alvo deste trabalho, é justificado porque favorece a conscientização dos mesmos sobre a importância do consumo de uma dieta apropriada e a adesão ao tratamento, prevenindo as complicações agudas e crônicas da doença e melhorando a qualidade de vida dessas pessoas. Assim, o presente estudo objetivou avaliar o alcance da intervenção nutricional sobre medidas antropométricas e glicemia de jejum dos participantes do Projeto de Atendimento Nutricional a Pacientes Diabéticos.

\section{MÉ TO D O S}

Este estudo foi conduzido de abril a dezembro de 2005 e envolveu todos os indivíduos portadores de diabetes mellitus participantes do Projeto de Atendimento Nutricional a Pacientes Diabéticos, da Universidade Federal de Viçosa, MG. A média mensal de consultas realizadas pelo projeto foi de 20 (Desvio-padrão - DP= 3). A amostra foi constituída de 42 indivíduos que atenderam aos seguintes critérios de inclusão: ter diagnóstico de diabetes mellitus de acordo com os critérios definidos pela American Diabetes Association, ter comparecido a, pelo menos, três atendimentos nutricionais individualizados, ter idade superior a 30 anos, ser residente do município de Viçosa e ter assinado o termo de consentimento para participar do estudo ${ }^{10}$. Não houve desistência ou necessidade de exclusão de nenhum dos participantes desta pesquisa. O protocolo do estudo foi aprovado pelo Comitê de Ética e Pesquisa da Universidade Federal de Viçosa (Protocolo n.40504654667).

Durante o período de estudo, os voluntários participaram de reuniões quinzenais e 
atendimentos ambulatoriais individualizados. Primeiramente, aplicou-se um questionário múltipla escolha, adaptado de Laguna et al. ${ }^{11}$, visando a identificar o nível de conhecimento dos integrantes em relação aos conceitos básicos sobre a doença, incluindo questões referentes ao diagnóstico e aos sintomas associados à enfermidade, importância do tratamento adequado e complicações resultantes do mau controle do diabetes. A partir daí, foram identificados os assuntos de menor domínio pelos participantes, os quais foram, então, abordados nas reuniões quinzenais. Alguns dos assuntos discutidos foram os seguintes: o que é o diabetes; importância da dieta, da prática de atividade física e do uso dos medicamentos prescritos para o controle da doença; principais complicações e morbidades associadas (retinopatia, pé-diabético, doenças cardiovasculares e renais etc.); além de dicas para a ingestão de uma dieta equilibrada e de cuidados com o corpo. Para estimular a adesão a tais reuniões, as atividades foram conduzidas por meio de técnicas de dinâmica de grupo, brincadeiras, debates e discussões, resultando, assim, na participação de todos os presentes.

Os atendimentos individualizados aconteceram uma vez por mês, sendo realizados nos consultórios da Divisão de Saúde da Universidade Federal de Viçosa. Na primeira consulta, foi preenchido um roteiro clínico-nutricional contendo dados pessoais, história clínica individual e familiar, hábitos gerais (fumo, prática de atividade física, consumo de bebidas alcoólicas e uso de medicamentos), anamnese e freqüência alimentar detaIhadas; avaliação bioquímica, antropométrica e de composição corporal. O questionário de freqüência alimentar continha 29 tipos de alimentos dos diversos grupos, com 10 possibilidades de resposta em relação ao consumo - diário; semanal $(6,5,4,3,2$ ou 1 vez na semana); quinzenal; mensal ou nunca. A partir das informações geradas por este instrumento, foi delineado o perfil de consumo inicial, para embasar a avaliação das mudanças alimentares que porventura ocorressem ao longo do período de intervenção.
Ao final de cada consulta, os pacientes receberam orientação dietética individualizada, de acordo com seus hábitos alimentares, comportamentais, dados bioquímicos, antropométricos e de composição corporal. O Guia Alimentar da Pirâmide dos Alimentos' ${ }^{12}$ foi utilizado como instrumento para educação nutricional, considerando os princípios básicos de uma dieta saudável: variedade, equilíbrio e moderação. Após a explicação das práticas alimentares recomendadas, o paciente foi incentivado a opinar sobre as providências a serem tomadas, objetivando melhorar sua própria alimentação. Foram prescritas dietas individualizadas, visando à manutenção ou obtenção de peso e composição corporal adequados, além do controle de eventuais alterações bioquímicas. Além disso, foram estabelecidas metas a serem atingidas pelo paciente até a próxima consulta. Durante o período de acompanhamento, registrou-se a evolução de cada participante.

Em todos os atendimentos, além da avaliação nutricional, verificou-se quais das metas estabelecidas que foram efetivamente alcançadas, ou seja, se houve melhora na qualidade da sua alimentação, conforme a proposta estabelecida pelo Guia da Pirâmide Alimentar. A seguir, os participantes tinham a oportunidade de expressar as dificuldades sentidas para seguir as orientações da(s) consulta(s) anterior(es), sendo, então, orientados em relação às possíveis formas para contornar tais dificuldades.

Os indivíduos foram pesados utilizando-se balança eletrônica digital, da marca Filizola, com capacidade de $150 \mathrm{~kg}$ e precisão de $50 \mathrm{~g}$, usando o mínimo de roupa possível e sem sapatos. 0 peso de cada participante do estudo foi avaliado em um mesmo horário pré-definido. A altura foi determinada utilizando-se um antropômetro vertical milimetrado, com extensão de $2 \mathrm{~m}$ e escala de $0,5 \mathrm{~cm}$. Os pacientes encontravam-se descalços, formando um ângulo de, aproximadamente, $45^{\circ}$ entre os pés, em posição ereta, olhando para o infinito, com as costas e a parte posterior dos joelhos encostados à parede ${ }^{13}$. $\mathrm{O}$ índice de massa 
corporal (IMC) dos indivíduos diabéticos foi calculado, relacionando o peso $(\mathrm{kg})$ e a altura (metros) ao quadrado, sendo avaliado segundo a classificação recomendada pela World Health Organization ${ }^{14}$.

Para classificar o risco de desenvolvimento de doenças cardiovasculares apresentado pelos indivíduos diabéticos, foi avaliada a medida da circunferência da cintura ${ }^{14,15}$. Essa medida foi aferida utilizando uma fita métrica inextensiva e inelástica, a $2,5 \mathrm{~cm}$ da cicatriz umbilical abaixo da costela, na linha média axilar, com o indivíduo em posição vertical9,16.

A composição corporal (quantidades e percentuais de gordura corporal total, massa livre de gordura e água corporal total) dos voluntários foi avaliada utilizando-se o método da bioimpedância elétrica vertical (BIA) (Biodynamics modelo 310). Esse aparelho utiliza a técnica clássica tetrapolar e freqüência fixa de $50 \mathrm{mHz}$. Os pacientes diabéticos eram posicionados em decúbito dorsal horizontal, com os braços e pernas levemente afastados do tronco. A composição corporal era obtida automaticamente, com auxílio de quatro eletrodos posicionados dois a dois, respectivamente, no dorso da mão e do pé. A equação do aparelho baseou-se nas medições de Lukaski et al. ${ }^{17}$.

Os pacientes foram orientados a realizar jejum de, pelo menos, duas horas antes da avaliação por bioimpedância, retirar meias, luvas ou objetos metálicos, não ingerir água nas horas precedentes ao exame, fazer o mínimo de atividade física e se abster do consumo de café no dia anterior ao teste. Indivíduos com uso de próteses de metal e/ou marcapasso foram afastados da avaliação. Além disso, orientou-se a micção com, pelo menos, 30 minutos de antecedência ao teste ${ }^{9,17}$.

Além das avaliações antropométricas e de composição corporal, os voluntários foram avaliados quanto à glicemia de jejum, que foi considerada normal entre 70 e $110 \mathrm{mg} / \mathrm{dL}^{18}$. Os exames laboratoriais foram solicitados trimestralmente. Os participantes foram orientados a não alterar o padrão alimentar nos dias anteriores à realização dos exames.

Os dados analisados no presente estudo foram obtidos a partir das fichas de atendimento nutricional e organizados em dois momentos diferentes, separados um do outro por um período equivalente a seis meses. Os dados iniciais correspondem àqueles referentes à primeira consulta $\mathrm{e}$ os dados finais, à mediana dos dados coletados durante os 6 meses de atendimento.

As análises estatísticas foram realizadas utilizando o software Sigma Stat versão 2.019. Para avaliar a alteração das medidas antropométricas, de composição corporal e dos níveis glicêmicos antes e depois do acompanhamento nutricional, foi utilizado o teste de Wilcoxon. Aplicou-se o teste de Mann-Whitney para comparar dois grupos independentes. Adotou-se nível de significância de $5 \%$.

\section{RES U L T A D O S}

$O$ atendimento nutricional individualizado foi oferecido a 42 pessoas, sendo $9,52 \%$ destas portadoras de diabetes do tipo 1 e 90,48\%, do tipo 2. A média de idade observada foi de 54,4 $(\mathrm{DP}=12,04)$ anos, sendo $59,52 \%(n=25)$ pacientes do sexo feminino e $40,48 \%$ ( $n=17)$ do masculino. Os pacientes receberam, em média, 4,38 atendimentos individualizados, com intervalo de 21,42 dias entre eles e freqüentaram cerca de $70,00 \%$ das reuniões quinzenais. O alcance do acompanhamento nutricional sobre o controle da doença foi avaliado considerando tanto as adequações antropométricas e bioquímicas, quanto a postura dos participantes nas reuniões, em função do nível das perguntas e das expressões faciais apresentadas pelos indivíduos, as quais permitiram perceber se o conteúdo estava sendo bem assimilado ou não.

Mais de 50,0\% dos voluntários associaram corretamente a manifestação de diabetes a uma alimentação inadequada, ao sedentarismo, à ocorrência de infecções, a fatores emocionais e 
genéticos. Evidenciou-se que os participantes tiveram mais dificuldade em responder os itens referentes aos aspectos metabólicos, como produção e função da insulina, influência da prática de atividade física no controle da doença e sintomatologia do diabetes (Tabela 1). Estes resultados contribuíram para a definição dos temas que foram abordados nos encontros em grupo.

A história clínica individual revelou que, aproximadamente, 90,0\% ( $n=38)$ dos pacientes apresentavam algum tipo de morbidade associada ao diabetes. A hipertensão arterial, referida em $71,4 \%$ casos $(n=30)$, foi a mais prevalente, seguida de obesidade ( $n=27 ; 64,3 \%$ ) e de outras doenças cardiovasculares, como a cerebrovascular e a isquemia do coração ( $n=18 ; 42,5 \%$ ).

Em relação aos hábitos de vida, no início do estudo observou-se um maior percentual de indivíduos sedentários ( $n=23 ; 54,76 \%$ ) que ativos. Por outro lado, o número de fumantes foi pequeno ( $n=6 ; 14,28 \%$ ) e a maioria dos participantes não consumia bebida alcoólica $(n=26 ; 61,90 \%)$. Ao

Tabela 1. Porcentagem (\%) de acertos às questões abordadas sobre conhecimento de diabetes a pacientes portadores da doença. Projeto Atendimento Nutricional a Pacientes Diabéticos. Viçosa (MG), 2005.

\begin{tabular}{|c|c|c|}
\hline Temas & $\begin{array}{c}\text { Pessoas que } \\
\text { responderam } \\
\text { corretamente }(n)\end{array}$ & $\%$ \\
\hline \multicolumn{3}{|l|}{$\begin{array}{l}\text { Possíveis fatores de risco para } \\
\text { a manifestação da doença }\end{array}$} \\
\hline Alimentação inadequada & 38 & 90,5 \\
\hline Sedentarismo & 36 & 85,7 \\
\hline Genética & 36 & 85,7 \\
\hline Emoção & 31 & 73,8 \\
\hline Infecção & 31 & 73,8 \\
\hline Alcoolismo & 25 & 59,5 \\
\hline Envelhecimento & 23 & 54,8 \\
\hline Insônia & 21 & 50,0 \\
\hline Fumo & 19 & 45,2 \\
\hline \multicolumn{3}{|c|}{ Noções gerais sobre o diabetes mellitus } \\
\hline Principais complicações & 33 & 78,6 \\
\hline Tratamento & 31 & 73,8 \\
\hline Prevenção & 29 & 69,0 \\
\hline Alimentação & 27 & 64,3 \\
\hline Atividade física & 25 & 59,5 \\
\hline Sintomas gerais & 25 & 59,5 \\
\hline Produção e função da insulina & 17 & 40,5 \\
\hline
\end{tabular}

final do estudo, verificou-se discreto aumento no número de praticantes de atividade física $(n=20$; $47,62 \%$ ), enquanto o total de fumantes e de consumidores de bebidas alcoólicas permaneceu inalterado.

O uso de hipoglicemiantes orais e/ou insulina foi relatado por 38 pessoas $(90,47 \%)$. Dos 42 indivíduos diabéticos estudados, 90,47\% ( $n=38)$ faziam associação entre o tratamento medicamentoso e o dietético e $42,85 \%(n=18)$ associavam o tratamento medicamentoso, o dietético e a prática de exercícios físicos.

Com relação ao número de refeições diárias realizadas pelos participantes, constatouse, ao final do estudo, um consumo médio entre 5 e 6 refeições diárias, em contraste com as 3 e 4 refeições referidas pelos indivíduos antes da intervenção. Pelas informações coletadas a partir das entrevistas aplicadas aos participantes, verificouse a ocorrência de mudanças na qualidade da dieta ingerida ao longo do estudo. Constatou-se o aumento do consumo de frutas, hortaliças e cereais integrais; maior preferência pela ingestão de leite desnatado; substituição do açúcar pelo adoçante artificial; diminuição do consumo per capita de sal e óleo vegetal.

O efeito das atividades desenvolvidas durante o estudo nos parâmetros antropométricos (peso corporal, IMC) e circunferência da cintura e de composição corporal (percentual de gordura corporal), de acordo com o sexo dos voluntários, pode ser observado na Figura 1. Tanto as mulheres $(4,7 \mathrm{~kg}$ em média), quanto os homens $(3,5 \mathrm{~kg}$ em média) apresentaram perda significante de peso $(p<0,05)$ ao final do estudo. Observou-se, ainda, redução estatisticamente significante $(p<0,05)$ no IMC das mulheres $\left(1,8 \mathrm{~kg} / \mathrm{m}^{2}\right.$ em média) e dos homens $\left(0,8 \mathrm{~kg} / \mathrm{m}^{2}\right.$ em média) voluntários. Ao final do estudo, constatou-se diferença significante no teor de gordura corporal, tanto para o sexo masculino $(p=0,045)$, quanto para o feminino $(p=0,039)$. De modo semelhante, verificou-se que os participantes apresentaram diminuição estatisticamente significante $(p=0,035)$ da glicemia de jejum em relação aos valores apresentados inicialmente (Figura 2). 

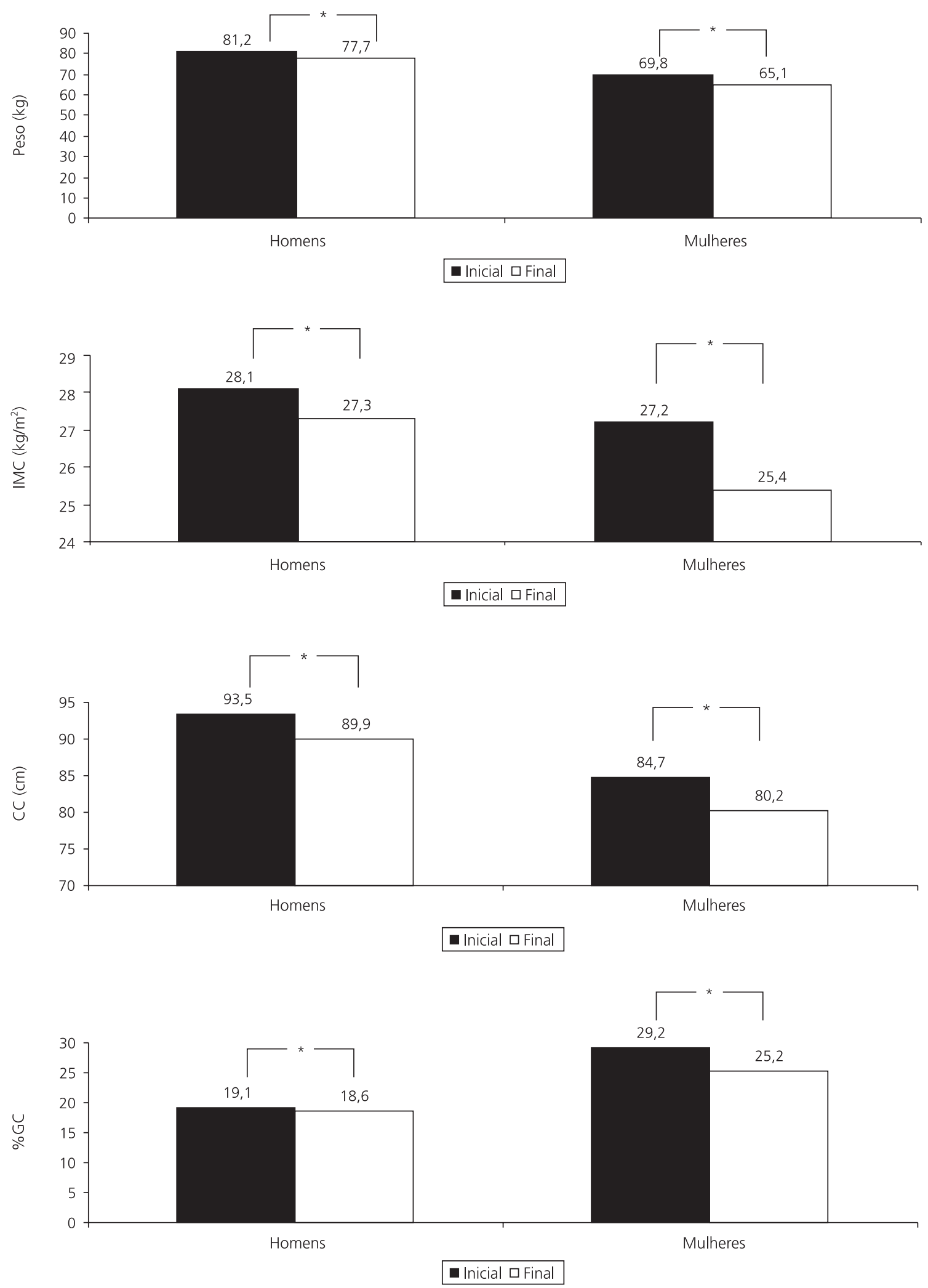

Figura 1. Valores referentes às medianas dos parâmetros antropométricos (peso, índice de massa corporal (IMC), circunferência da cintura - CC) e de composição corporal (percentual de gordura corporal - \%GC) de pacientes diabéticos, obtidos ao início do estudo e após 6 meses de acompanhamento nutricional ( $\left.{ }^{*} p<0,05\right)$. Viçosa (MG), 2005. 


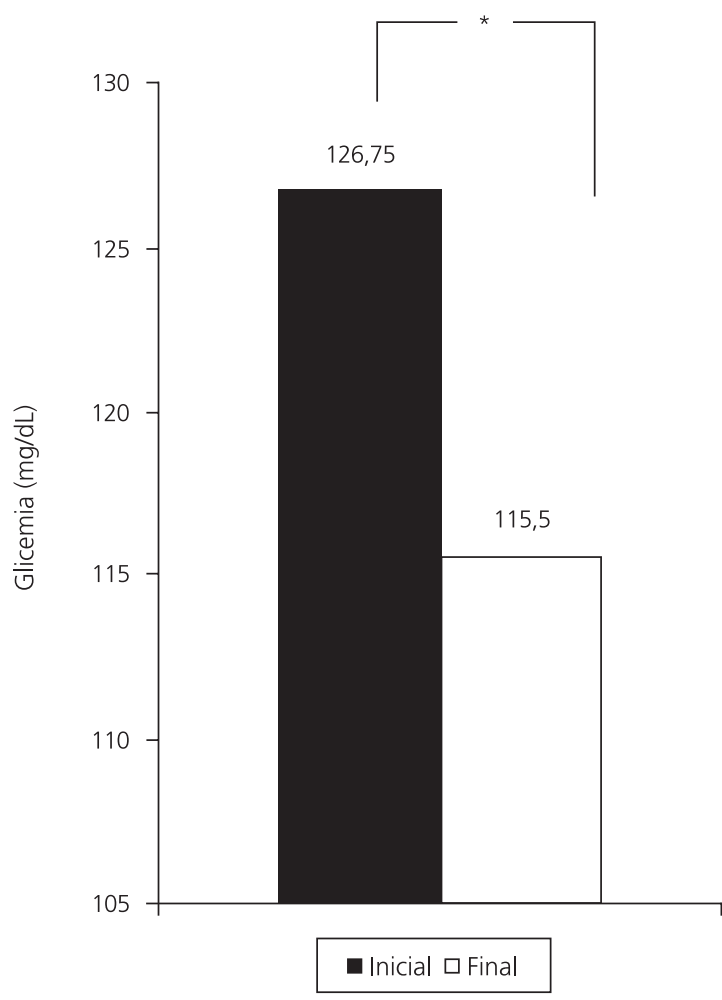

Figura 2. Valores referentes às medianas de glicemia de jejum de pacientes diabéticos, obtidos ao início do estudo e após 6 meses de acompanhamento nutricional ( $\left.{ }^{*} p=0,035\right)$. Viçosa (MG), 2005.

A classificação obtida da circunferência da cintura apontou que $80,0 \%$ das mulheres $(n=20)$ e $70,5 \%$ dos homens ( $n=12)$ apresentavam esta medida aumentada antes da intervenção, de acordo com os critérios estabelecidos pela World Health Organization ${ }^{14}$. Entretanto, houve redução significante deste parâmetro antropométrico nos dois sexos ao final do estudo (CC mediana de 80,2 nas mulheres e 89,9 nos homens), resultando, assim, na redução do risco de desenvolvimento de doenças cardiovasculares.

Para avaliar a eficácia da intervenção, empregou-se a comparação de medianas (testes não-paramétricos), já que a análise estatística indicou que as variáveis não apresentavam distribuição normal. Entretanto, somente para termos comparativos, também foram incluídos na Tabela 2 os valores das médias e desvios-padrão observados para cada uma das variáveis, bem como a amplitude das mesmas.

\section{I S CUSS Ã O}

Quase todos os participantes $(90,48 \%)$ deste estudo eram diabéticos do tipo 2 e apresentavam em média 54,4 ( $D P=12,04)$ anos. $O$ maior percentual de mulheres $(59,52 \%)$ evidenciado pode ser explicado pela grande preocupação das mesmas com a saúde e, conseqüentemente,

Tabela 2. Dados antropométricos (peso, índice de massa corporal (IMC), circunferência da cintura - CC) e de composição corporal (percentual de gordura corporal - \%GC) de pacientes diabéticos. Projeto Atendimento Nutricional a Pacientes Diabéticos, antes e após 6 meses de intervenção nutricional. Viçosa (MG), 2005.

\begin{tabular}{|c|c|c|c|c|c|c|c|c|c|c|c|}
\hline \multirow{2}{*}{ Variável } & \multicolumn{2}{|c|}{ Mediana } & \multicolumn{2}{|c|}{ Mínimo } & \multicolumn{2}{|c|}{ Máximo } & \multicolumn{2}{|c|}{ Média } & \multicolumn{2}{|c|}{ Desvio-padrão } & \multirow{2}{*}{$\begin{array}{l}\text { Valores } \\
\text { de } p^{*}\end{array}$} \\
\hline & Antes & Depois & Antes & Depois & Antes & Depois & Antes & Depois & Antes & Depois & \\
\hline \multicolumn{12}{|c|}{ Sexo masculino } \\
\hline Peso (kg) & 81,2 & 77,7 & 68,4 & 68,0 & 100,3 & 97,7 & 84,4 & 82,9 & 22,6 & 21,0 & 0,021 \\
\hline IMC $\left(\mathrm{kg} / \mathrm{m}^{2}\right)$ & 28,1 & 27,3 & 21,0 & 21,0 & 34,6 & 33,8 & 27,8 & 27,4 & 9,6 & 9,1 & 0,032 \\
\hline$C C(\mathrm{~cm})$ & 93,5 & 89,9 & 70,4 & 70,3 & 105,0 & 103,2 & 87,7 & 86,8 & 24,5 & 23,3 & 0,036 \\
\hline$\% G C$ & 19,1 & 18,6 & 17,1 & 17,1 & 29,1 & 29,0 & 23,1 & 23,1 & 8,5 & 8,4 & 0,045 \\
\hline \multicolumn{12}{|c|}{ Sexo feminino } \\
\hline Peso (kg) & 69,8 & 65,1 & 51,2 & 49,7 & 82,3 & 81,2 & 66,8 & 65,5 & 22,0 & 22,3 & 0,037 \\
\hline IMC $\left(\mathrm{kg} / \mathrm{m}^{2}\right)$ & 27,2 & 25,4 & 19,7 & 19,5 & 32,5 & 30,4 & 26,1 & 25,0 & 9,1 & 7,7 & 0,022 \\
\hline$C C(\mathrm{~cm})$ & 84,7 & 80,2 & 69,7 & 68,7 & 97,4 & 96,7 & 83,6 & 82,7 & 19,6 & 19,8 & 0,030 \\
\hline$\% \mathrm{GC}$ & 29,2 & 25,2 & 23,0 & 21,0 & 34,0 & 33,0 & 28,5 & 27,0 & 7,8 & 8,5 & 0,039 \\
\hline
\end{tabular}

*Considerando os valores das medianas. 
maior busca por informações e serviços de atenção ao diabetes ${ }^{7}$. Segundo alguns autores, a ocorrência de diabetes mellitus é maior no sexo feminino, porém essa diferença não é estatisticamente significante ${ }^{20,21}$. Observa-se que a diferença na freqüência entre sexos constitui, mais do que um reflexo de potenciais genéticos, um produto da presença de diversos fatores ambientais, que variam para cada sociedade. Portanto, os maiores índices de diabetes no sexo feminino têm sido associados a tais fatores, visto que o sexo em si não é considerado fator de risco para a doença ${ }^{22,23}$.

Ao analisarem o comportamento clínicoepidemiológico do diabetes mellitus em um município de Cuba, Laguna et al. ${ }^{11}$ obtiveram resultados semelhantes aos verificados no presente trabalho em relação ao desconhecimento dos portadores de diabetes sobre a doença. Segundo os autores daquele estudo, os resultados foram atribuídos à falta de programas estatais intervencionistas, os quais poderiam ser úteis no controle da doença.

No estudo atual, observou-se a importância de enfatizar, utilizando linguagem apropriada, os aspectos referentes à ação da insulina no organismo, os sintomas gerais associados ao mau controle glicêmico, bem como o efeito benéfico resultante da ingestão de dieta adequada e da prática regular de exercícios físicos para o controle da doença. Esses aspectos foram especialmente abordados nas reuniões em grupo, favorecendo o bom entrosamento entre a equipe executora do estudo e os participantes da pesquisa, contribuindo para a aprendizagem dos assuntos abordados. De acordo com estudo de Maia \& Araújo ${ }^{24}$, o uso de dinâmicas de grupo, associadas a lazer e cultura, proporcionou um maior crescimento intelectual e pessoal dos indivíduos, resultando em mais segurança e melhor aceitação das doenças apresentadas.

Pesquisadores apontam que, entre as variáveis que mais dificultaram a adesão às recomendações nutricionais por pacientes diabéticos, destacam-se as dificuldades financeiras para aquisição dos tipos de alimentos mais apropriados ao tratamento, a influência da família no consumo de alimentos, além da falta de clareza na análise da relação custo-benefício entre adesão às orientações e os fatores de risco associados às descompensações glicêmicas ${ }^{25,26}$.

O diabetes se associa ao sedentarismo e à manifestação de doenças crônicas não transmissíveis, como obesidade, hipertensão arterial, doenças cardiovasculares $22,23,27,28$. A prática de atividades físicas tem sido recomendada aos pacientes diabéticos, por favorecer a redução dos níveis glicêmicos, aumentando a sensibilidade insulínica e melhorando a tolerância à glicose, já que o exercício promove um aumento do turnover da insulina por maior captação hepática e melhor sensibilidade dos receptores periféricos ${ }^{22,27}$. Ao final deste estudo, mais da metade dos indivíduos diabéticos pesquisados ainda não realizava exercícios físicos regularmente, o que pode interferir negativamente na saúde dos mesmos. Resultado semelhante foi observado por Laguna et al. ${ }^{11}$, os quais ressaltam a importância da adoção de medidas que estimulem a prática regular de atividades físicas por pacientes diabéticos, especialmente os do tipo 2, para a obtenção do controle glicêmico e melhora da qualidade de vida.

A obesidade e o sobrepeso têm sido apontados como fatores que favorecem a manifestação do diabetes do tipo $2^{7,22,29}$. A relação entre diabetes do tipo 2 e obesidade tem sido atribuída ao aumento da resistência insulínica em indivíduos com grandes depósitos de gordura, principalmente na região central ${ }^{29}$. Sabe-se que a circunferência da cintura é o parâmetro antropométrico mais adequado para avaliar o acúmulo de gordura na região central ${ }^{14}$. No início do estudo, $76,2 \%$ dos participantes considerados diabéticos do tipo 2 apresentavam circunferência da cintura acima do recomendado. Durante o período de estudo, os participantes apresentaram redução significante $(p=0,038)$ deste parâmetro. Estes resultados têm um impacto positivo sobre o aumento da sensibilidade insulínica e a redução do risco de manifestação de doenças cardiovasculares ${ }^{14}$. 
Os valores de IMC têm sido associados diretamente aos valores de glicemia sanguínea. O uso desse índice é simples, correlaciona-se com outras medidas corporais e tem sido reconhecido como o indicador que, isoladamente, permite o melhor diagnóstico da situação nutricional de adultos no plano coletivo ${ }^{20}$. Em estudo conduzido por Cabrera-Pivaral et al. ${ }^{20}$, a diminuição do IMC correlacionou-se positivamente com a queda da glicemia em mexicanos portadores de diabetes mellitus tipo 2 submetidos a um programa educativo, no qual aspectos relacionados à ingestão de uma dieta saudável foram abordados. Apesar de os valores de IMC terem apresentado redução significante no presente estudo, este valor ainda se encontra ligeiramente elevado em relação à recomendação ${ }^{14}$. Estudo comparativo entre portadores de diabetes mellitus do tipo 2 que participavam ou não de grupo educativo, mostrou redução dos níveis de glicemia e melhor conhecimento sobre o diabetes entre os participantes de atividades educativas em saúde ${ }^{25}$.

Dados da literatura indicam que as mulheres portadoras de diabetes apresentam maior risco cardiovascular que os homens com esta mesma condição, em função do maior percentual de gordura corporal encontrado entre as primeiras ${ }^{30}$.

Estudo prospectivo realizado por Corrêa et al. ${ }^{31}$ evidenciou que medidas antropométricas e de composição corporal, como o IMC, o percentual de gordura corporal, a circunferência abdominal e a relação cintura-quadril se associaram diretamente ao controle glicêmico no diabetes mellitus do tipo 2. A maior adequação de tais variáveis não só neste, como também naquele estudo, pode, portanto, contribuir para a melhora na qualidade de vida dos indivíduos diabéticos.

Tem sido comprovado que a hipertensão arterial sistêmica por si só acarreta um aumento da freqüência dos acidentes coronários e cerebrovasculares na população em geral ${ }^{32}$. Assim, a alta freqüência de hipertensão arterial sistêmica $(71,4 \%)$, pode ter sido responsável pela elevada ocorrência de doenças cardiovasculares observada $(42,5 \%)$ entre os indivíduos diabéticos deste estudo. No entanto, o controle adequado dos níveis glicêmicos pode resultar na redução da prevalência de hipertensão arterial sistêmica entre os indivíduos diabéticos ${ }^{28}$.

Os resultados deste trabalho demonstraram que o consumo de álcool e o hábito de fumar não eram freqüentes entre os voluntários. Em geral, tal fato é atribuído à grande procura dos pacientes diabéticos pelos serviços de saúde, nos quais são estimulados a adotar hábitos de vida saudáveis, incluindo a suspensão do tabagismo e do etilismo ${ }^{28}$.

\section{O N CLUS Ã O}

Os resultados obtidos no presente estudo sugerem que as atividades desenvolvidas durante a execução do mesmo podem ter contribuído para a adequação das medidas antropométricas, de composição corporal e da glicemia de jejum dos participantes. A perda ponderal observada, provavelmente, contribuiu para o aumento da sensibilidade insulínica, favorecendo para a redução da glicemia de jejum dos indivíduos diabéticos ${ }^{1,22}$. Entretanto, a mediana $(115,5 \mathrm{mg} / \mathrm{dL})$ da glicemia de jejum observada ao final do estudo ainda se encontra na faixa de risco, devendo ser reduzida. Além disso, apesar da tendência à adequação dessa medida bioquímica, é necessária a adesão ao tratamento proposto por um período de tempo maior, para que as alterações se tornem ainda mais evidentes.

\section{OLABORADORES}

J.M. Geraldo colaborou no planejamento das atividades desenvolvidas durante o estudo, na divulgação do projeto Atendimento Nutricional a Pacientes Diabéticos, no planejamento das reuniões com os sujeitos, no desenvolvimento das técnicas de dinâmica de grupo, na realização de atendimento nutricional individualizado, na análise e na interpretação dos resultados. R.C.G. ALFENAS coordenou o projeto, planejou as atividades desenvolvidas, supervionou e acompanhou as atividades, análisou e interpretou os resultados. R.D.M. ALVES e V.F. SALLES 
planejaram e participaram das reuniões com os sujeitos, desenvolveram técnicas de dinâmica de grupo, realizaram o atendimento nutricional individualizado. V.M.V. QUEIROZ e M.C.B. BITENCOURT planejaram e participaram das atividades em grupo, especialmente no que se refere às técnicas de preparo de receitas culinárias.

\section{REFERÊ N CIAS}

1. American Diabetes Association. Standards of medical care in diabetes. Diabetes Care. 2005; 28(Suppl 1):S4-S36.

2. World Health Organization. Diet, nutrition and the prevention of chronic diseases. Report WHO Consultation. Geneva: WHO; 2003. Technical Report Series, 916.

3. Sociedade Brasileira de Diabetes. Atualização brasileira sobre diabetes. Rio de Janeiro: Diagraphic; 2005.

4. Miyar LO. Impacto de un programa de promoción de la salud aplicado por enfermería a pacientes diabéticos tipo 2 en la comunidad. Rev Latinoam Enferm. 2003; 11(6):713-9.

5. Amos AF, Mccarty DJ, ZimmeT P. The rising global burden of diabetes and its complications: estimates and projections to the year 2010. Diabet Med. 1997; 14(Suppl 5):S1-S5.

6. Guimarãs FPM, Takayanagui AMM. Orientações recebidas dos serviços de saúde por pacientes para o tratamento do portador de diabetes mellitus tipo 2. Rev Nutr. 2002; 15(1):37-44.

7. Batista MCR, Priore SE, Rosado LEFPL, Tinôco ALA, Franceschini SCC. Avaliação dos resultados da atenção multiprofissional sobre o controle glicêmico, perfil lipídico e estado nutricional de diabéticos atendidos em nível primário. Rev Nutr. 2005; 18(2):219-28.

8. American Diabetes Association. Nutritional principals and recommendations in diabetes. Diabetes Care. 2004; 27(Suppl 1):S36-S46.

9. Duarte ACG, Castellani FR. Semiologia nutricional. Rio de Janeiro: Axcel Books do Brasil; 2002.

10. American Diabetes Association. Standards of medical care in diabetes - 2006. Diabetes Care. 2006; 29(Suppl 1):S4-S42.

11. Laguna LS, Segura OS. Comportamiento clínicoepidemiológico de la diabetes mellitus en el municipio Jobabo. Hospital "14 de Junio". Corr Med Cient Holg. 2005; 9(1):11-6.
12. Food Guide Piramyd. A guide to daily food choices. Washington (DC): US Department of Agriculture, Human Nutrition Information Service; 1992. Home and Garden Bulletin n.232.

13. Bray GA, Gray DS. Obesity I: phathogenesis. West J Med. 1988; 149(4):429-41.

14. World Health Organization. Obesity: preventing and managing the global epidemic. Report WHO Consultation. Geneva; 2000. Technical Report Series, 894.

15. Kissebah $\mathrm{AH}$. Central obesity: measurement and metabolic effects. Diabetes Rev. 1997; 5(1):8-20.

16. Lean MEJ, Han TS, Morrison CE. Waist circumference as a measure for indicating need for weight management. BMJ. 1995; 311(Suppl 1):158-61.

17. Lukaski HC, Johnson PE, Bolonchuk WW, Lykken GI. Assesment of fat-free mass using bioelectrical impedance measurements of the human body. Am J Clin Nutr. 1985; 41(4):810-7.

18. Sociedade Brasileira de Diabetes. Consenso brasileiro sobre diabetes: diagnóstico e classificação e tratamento do diabetes mellitus Tipo 2; 2002; Rio de Janeiro: Diagraphic; 2003.

19. Fox E, Kuo J, Tilling L, Ulrich C. User's manual Sigma Stat: Statistical software for Windows [computer program]. Version 2.0. Berlim: Jandel; 1994.

20. Cabrera-Pivaral CE, González-Pérez G, Vega-López MG, Arias-Merino ED. Impacto de la educación participativa en el indice de masa corporal y glicemia en individuos obesos con diabetes tipo 2. Cad Saúde Pública. 2004; 20(1):275-81.

21. Hu FB, Manson JE, Stampfer MJ, Colditz G, Liu S, Solomon CG, et al. Diet, lifestyle and the risk of type 2 diabetes mellitus in women. N Engl J Med. 2001; 345(11):790-7.

22. Barceló, A. No non-insulin-dependent diabetes mellitus (NIDDM). Monograph Series on agingrelated diseases: VIII. Chronic Dis Can. 1997; 17(1): 10-29.

23. Goldenberg P, Schenkman S, Franco LJ. Prevalência de diabetes mellitus: diferenças de gênero e igualdade entre os sexos. Rev Bras Epidemiol. 2003; 1(6):18-28.

24. Maia FFR, Araújo LR. Projeto "Diabetes Weekend": proposta de educação em diabetes mellitus tipo 1. Arq Bras Endocrinol Metab. 2002; 46(5): 566-73.

25. Cazarini RP, Zanetti ML, Ribeiro KP, Pace $A E$, Foss MC. Adesão a um grupo educativo de pessoas portadoras de diabetes mellitus: porcentagem e causas. Medicina (Ribeirão Preto). 2002; 35(1): 142-50. 
26. Silva I, Ribeiro-Pais J, Cardoso H, Ramos H. Qualidade de vida e complicações crônicas da diabetes. Anál Psicol. 2003; 2(12):185-94.

27. Gomes VB, Siqueira KS, Sichieri R. Atividade física em uma amostra probabilística da população do Município do Rio de Janeiro. Cad Saúde Pública. 2001; 17(4):969-76.

28. Schaan BD, Harzheim E, Gus I. Perfil de risco cardíaco no diabetes mellitus e na glicemia de jejum alterada. Rev Saúde Pública. 2004; 38(4):529-36.

29. Pereira LO, Francischi RP, Lancha Jr AH. Obesidade: hábitos nutricionais, sedentarismo e resistência à insulina. Arq Bras Endocrinol Metab. 2003; 47(2):111-27.

30. Wei M, Gaskill SP, Haffner SM, Stern MP. Effects of diabetes and level of glycemia on all-cause and cardiovascular mortality: the San Antonio heart study. Diabetes Care. 1998; 21(7):1167-72.

31. Corrêa FHS, Taboada GF, Júnior CRMA, André AM, Clemente ELS, Fuks AG, et al. Influência da gordura corporal no controle clínico e metabólico de pacientes com diabetes mellitus tipo 2. Arq Bras Endocrinol Metab. 2003; 47(1):62-8.

32. Imbert WMG, Diaz YP, Llorente MS, Lima IL. Comportamiento de la educación diabetológica en el Hospital Militar Doctor Mario Muñoz Monroy del municipio Matanzas. Rev Cienc Méd Cuba. 2004; 216(1):56-62.

Recebido em: 11/4/2006

Versão final reapresentada em: 10/11/2006 Aprovado em: 15/2/2008 